\title{
Polyhedral Voronoi diagrams for additive manufacturing
}

\author{
JONÀS MARTÍNEZ, SAMUEL HORNUS, HAICHUAN SONG, and SYLVAIN LEFEBVRE, Université de Lor- \\ raine, CNRS, Inria, LORIA, F-54000 Nancy, France
}
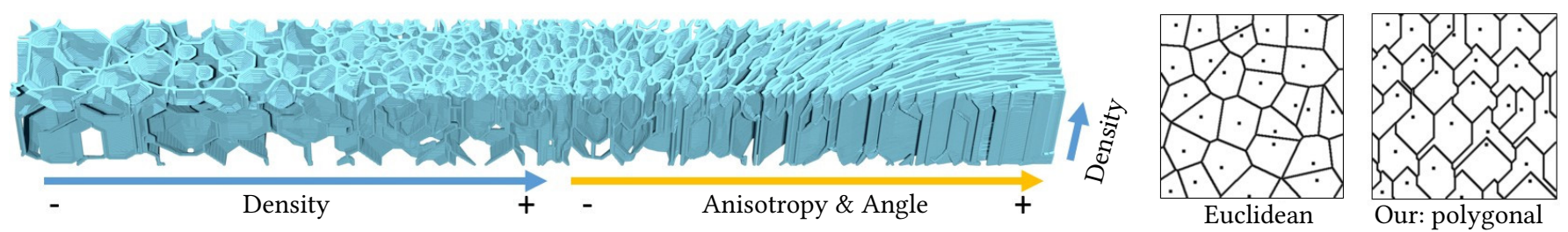

Fig. 1. Left: We propose a novel type of microstructures well suited for fabrication on continuous deposition processes such as fused filament fabrication. Our microstructures afford for a wide range of elastic behaviors, from isotropic to orthotropic. As illustrated their geometry can be spatially graded. Right: We model the foams as Voronoi diagrams of a special kind, which contrary to Euclidean Voronoi diagrams produce geometry enforcing all fabrication constraints.

A critical advantage of additive manufacturing is its ability to fabricate complex small-scale structures. These microstructures can be understood as a metamaterial: they exist at a much smaller scale than the volume they fill, and are collectively responsible for an average elastic behavior different from that of the base printing material making the fabricated object lighter and/or flexible along specific directions. In addition, the average behavior can be graded spatially by progressively modifying the microstructure geometry.

The definition of a microstructure is a careful trade-off between the geometric requirements of manufacturing and the properties one seeks to obtain within a shape: in our case a wide range of elastic behaviors. Most existing microstructures are designed for stereolithography (SLA) and laser sintering (SLS) processes. The requirements are however different than those of continuous deposition systems such as fused filament fabrication (FFF), for which there is currently a lack of microstructures enabling graded elastic behaviors.

In this work we introduce a novel type of microstructures that strictly enforce all the requirements of FFF-like processes: continuity, self-support and overhang angles. They offer a range of orthotropic elastic responses that can be graded spatially. This allows to fabricate parts usually reserved to the most advanced technologies on widely available inexpensive printers that also benefit from a continuously expanding range of materials.

CCS Concepts: • Computing methodologies $\rightarrow$ Shape modeling;

Additional Key Words and Phrases: Voronoi diagram, 3D printing, additive manufacturing

\section{ACM Reference Format:}

Jonàs Martínez, Samuel Hornus, Haichuan Song, and Sylvain Lefebvre. 2018. Polyhedral Voronoi diagrams for additive manufacturing. ACM Trans. Graph. 37, 4, Article 129 (August 2018), 15 pages. https://doi.org/10.1145/ 3197517.3201343

Permission to make digital or hard copies of all or part of this work for personal or classroom use is granted without fee provided that copies are not made or distributed for profit or commercial advantage and that copies bear this notice and the full citation on the first page. Copyrights for components of this work owned by others than the author(s) must be honored. Abstracting with credit is permitted. To copy otherwise, or republish, to post on servers or to redistribute to lists, requires prior specific permission and/or a fee. Request permissions from permissions@acm.org.

(c) 2018 Copyright held by the owner/author(s). Publication rights licensed to ACM. 0730-0301/2018/8-ART129 \$15.00

https://doi.org/10.1145/3197517.3201343

\section{INTRODUCTION}

Novel capabilities of additive manufacturing processes are challenging the traditional separation between shape and material. Tool accessibility and mold extraction constraints no longer apply, and shapes with intricate internal details can be fabricated. These internal microstructures modify the large-scale material properties, making parts lighter, porous or flexible and resilient. In addition, these microstructures can be spatially graded: the same object may be rigid where external stresses apply, and lighter in other regions.

This has spawned an intense research effort towards the geometric design of microstructures that can be embedded into volumes while triggering varying properties. The microstructures are typically considered as a metamaterial: at large-scale they behave equivalently to a uniform material characterized by a homogeneous elasticity tensor. This average behavior can be analyzed, abstracting away the fine-scale details, in a process called numerical homogenization. In addition, the microstructures are often parametric: the geometry of their fine scale details is controlled through high-level parameters, such as thickness or orientation. These parameters impact the large-scale elastic behavior, and thus the observed elasticity tensor can be controlled by manipulating these parameters directly. The main interest in using such parametric metamaterials is that their elasticity can be spatially controlled - graded - by a control field, varying mechanical properties within the shape volume. The control field can be either manually painted through a dedicated user interface [Ion et al. 2016] or automatically computed through, e.g. topology optimization [Zhu et al. 2017].

In this paper we focus on defining a novel class of parametric metamaterials. A key challenge - besides triggering the desired elastic behavior - is to enforce constraints due to the additive manufacturing processes. Most existing works on the topic have focused on processes such as selective laser sintering (SLS) and stereolithography (SLA). These technologies have different constraints than the widely available and inexpensive fused filament fabrication (FFF) 3D printers. In particular, most recent works produce geometries exhibiting truss structures (detailed in Section 2). After slicing, these structures produce many isolated ellipses in each slice. These are extremely challenging to print on FFF systems, where continuity 
of deposition, low overhang angles, and support from below are strict requirements (for examples of actual trials we recommend the online article by Zhou [2015])

This is especially unfortunate, as FFF is not only widely available but allows to fabricate in a wide spectrum of materials, from inexpensive plastics, food-safe materials, nylons, to metal-polymer compounds and carbon fiber reinforced materials (see filament by e.g. Proto-Plasta). In addition, other technologies such as wire arc metal printing ${ }^{1}$ and contour crafting ${ }^{2}$ share the same constraints. These constraints are very restrictive and have so far prevented the development of metamaterials for these processes.

Contributions. Our paper aims at filling this gap, defining a novel parametric metamaterial with the following properties:

- closed-cell, stochastic foam-like geometry readily printable on FFF-like systems, with strictly enforced overhang angles and that is everywhere supported from below,

- density and anisometry controllable through user provided fields that can vary arbitrarily,

- defines orthotropic materials that can be freely oriented in the plane orthogonal to the fabrication direction,

- a purely procedural computation that scales to arbitrarily large shapes.

This is made possible by defining procedural Voronoi diagrams based on polyhedral distances. We identify a class of parameterized polyhedral distances providing guarantees on the maximum overhang angles, and ensuring that all deposited material is supported from below. These constraints remain enforced when varying density and orientation parameters. We detail the procedural generation of our foams, analyze their elastic behavior and the link between the underlying parameters and the obtained elasticity tensors, and produce a variety of results demonstrating applications.

\section{RELATED WORK}

Researchers in additive manufacturing have recognized the need for internal fill patterns early on [McMains et al. 2000]. Indeed, by avoiding densely filling an interior, the print time and material use are greatly reduced. Thus, early infill patterns were essentially targeted at reducing material density, while being fast to generate and efficient to fabricate. Typical patterns for continuous deposition encompass parallel lines with varying spacing, but more elaborate patterns have been devised in an effort to achieve better strength to weight ratios [Livesu et al. 2017].

In recent years, the focus has shifted to producing infill patterns with controllable mechanical properties. Most approaches cast the problem as a two-scale optimization [Zhu et al. 2017]. A parametric metamaterial is defined either as a set of (parametric) periodic microstructures [Panetta et al. 2017, 2015; Schumacher et al. 2015] or as a random process producing geometry [Martínez et al. 2016; Martínez et al. 2017]. The link between microstructure parameters and their average elastic behavior is analyzed, defining a material space. Each entry of the material space relates a choice of structure

\footnotetext{
${ }^{1}$ See $e . g$. https://waammat.com/about/waam

${ }^{2}$ See https://en.wikipedia.org/wiki/Contour ${ }_{\mathrm{c}}$ rafting
}

parameters to an elasticity tensor. A user or an algorithm can directly work at a coarser scale and specify the desired elastic behavior in different regions of space. This is translated into microstructure parameters used to produce a final geometry. The definition of the geometry is often procedural, such that it is instantiated only when sent to the printer, slice by slice [Pasko et al. 2011; Vidimče et al. 2013]. Our work is inscribed in this direction of research.

Most results in this area produce microstructures that are fabricable only with SLS/SLA, which have constraints different from FFF. The challenges in designing infill patterns for continuous deposition are threefold. First, material can only be deposited on top of already solidified layers: it is not possible to start a new feature in 'mid-air'. Similarly, while slanted walls can be fabricated, there is a limit to the maximum overhang angle (typically around $45 \mathrm{de}$ grees, varies with layer and deposition thicknesses). Second, the extrusion process should be interrupted as little as possible, as each start/stop produces small deposition defects. Third, the deposition head is typically a relatively heavy device, and acceleration rates are limited by mechanical capabilities.

The first constraint (overhangs, support) relates to the geometry of the patterns and requires defining self-supporting geometry (e.g. not requiring auxiliary support for fabrication). The two other constraints (continuity, acceleration) relate to deposition efficiency and quality. In particular, truss structures which print well on SLA/SLS are ill-suited for continuous deposition due to the last two constraints: they require printing many small discs spread throughout each layer. This triggers many start/stop and abrupt speed changes - the low acceleration limiting the maximum reachable speed. As a consequence, the beams have lower quality and print slower than their volumes would indicate. Unsurprisingly, most patterns for FFF are comprised of as long as possible continuous lines.

Nevertheless, recent research has considered improved infill patterns for continuous deposition. Leary et al. [2013] advocate for the use of self-supported spatial tessellations, considering maximum overhang angles. Wu et al. [2016] explore how sub-dividable rhombic infill patterns [Lefebvre 2015] can be optimized for rigidity. Lee and Lee [2017] optimize similar patterns to produce parts that are as empty as possible - while producing self-supported structures. Hornus et al. [2016], Xie et al. [2017] and Wang et al. [2017] consider how to maximally carve parts while producing self-supported inner cavities. Lu et al. [2014] optimize the positions of the point sites of a Voronoi diagram to strengthen an object. Interestingly, a soluble support material had to be used within the cells (page 6, paragraph Physical test of [Lu et al. 2014]) - that is because usual Voronoi diagrams cannot form self-supported structures (see Section 3). Lee et al. [2018] pack many ellipses within a volume interior to obtain a self-supported interior fill.

However, none of these techniques afford for the definition of a material space, in the same sense as the microstructures employed with SLS/SLA [Martínez et al. 2016; Panetta et al. 2015; Schumacher et al. 2015]. One exception is the work of Martinez et al. [2017] on orthotropic foams, where orthotropic 2D patterns are fabricated on FFF printers. However, this only applies to 2D patterns extruded vertically, not 3D structures. Subdivisible infills [Medeiros e Sá et al. 2015; Wu et al. 2016] come close, but these cannot produce smooth 
spatial variations in orientation and density due to their underlying regular nature. We discuss them in more details and provide elements of comparison in Section 5.5.

Our work aims at introducing infill patterns which are well suited for fabrication by continuous deposition, can be graded spatially, and provide a wide material space for design.

\section{OVERVIEW}

The microstructures we explore belong to the family of closed-cell foams. They are defined as a stochastic process, which parameters drive the small-scale material arrangement, and ultimately the average elastic behavior.

Our foams are defined as the thickening of the facets of Voronoi diagrams of a special kind. We give below some more specific background on the use of Voronoi diagrams to represent foams and microstructures, explain the challenges and give some intuition on our technique. The precise definition and analysis of our microstructures are given in Section 4.

\subsection{Voronoi foams in additive manufacturing}

In material science, naturally occurring cellular solids are often idealized as sets of Voronoi edges (open-cell) or facets (closed-cell) [Gibson and Ashby 1999]. These have been well studied as they exhibit desirable properties such as an isotropic elastic behavior [Luxner et al. 2007] with a quasi-linear link between density and Young's modulus [Gibson and Ashby 1999; Roberts and Garboczi 2002].

Voronoi open-cell foams are used for fabrication on SLA/SLS processes, as they form fully connected truss networks well suited to resin and powder solidification. They are amenable to efficient procedural synthesis [Martínez et al. 2016]. Open-cell foams are however not very suitable for continuous deposition, due to the constraints mentioned in Section 2. For continuous deposition, closedcell foams are more promising: they form walls that would afford for continuous deposition and are also known to exhibit good mechanical behaviors - in fact, for the same volume closed-cell foams are more rigid than open-cell foams (see [Gibson and Ashby 1999, Formulas 5.6, 5.13] and [Sigmund et al. 2016]). The reason closedcell foams are not used with SLA/SLS is that they would trap non solidified material in closed cells. This, however, is not an issue on FFF-like processes.

Unfortunately, as we discuss next, the usual Voronoi diagrams do not define self-supporting closed-cell structures. Besides, their elastic behavior would remain limited to isotropic elasticity, while we seek a wider material space.

\subsection{Challenges of usual Voronoi diagrams}

We now discuss why usual (Euclidean) Voronoi diagrams cannot be readily used. Let us consider such a diagram defined from a set of point sites $s_{i} \in S$. Each Euclidean Voronoi cell is defined by:

$$
V_{P}\left(s_{i}\right)=\left\{p \in \mathbb{R}^{3}:\left\|s_{i}-p\right\|_{2}<\left\|s_{j}-p\right\|_{2}, \forall s_{i} \neq s_{j} \in S\right\}
$$

The closed-cell foam is formed by thickening the facets of the diagram. Figure 2 shows 2D examples where facets are edges. In these side views, the build direction goes upward. As can be seen in the Euclidean diagram (leftmost), the facets/edges can take an arbitrary orientation and many of them violate the overhang constraint: they cannot be printed on continuous deposition systems. In addition, when the point sites are uniformly distributed, the cells tend to be isotropic, which prevents the emergence of anisotropic elastic behaviors (see [Martínez et al. 2017]).

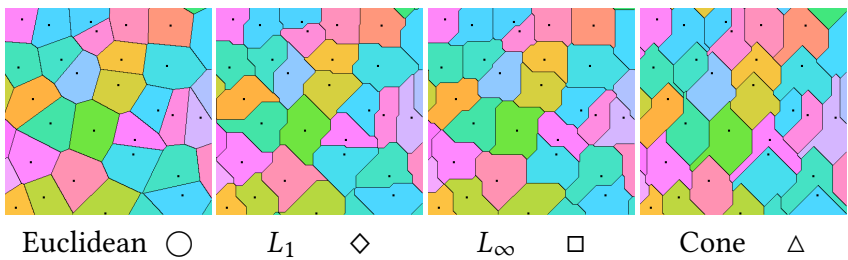

Fig. 2. The Voronoi diagram of a set of points under various distances, with their unit ball drawn next to the name.

3.2.1 Changing the distance. Voronoi diagrams can be defined using different distances. Figure 2 shows the diagram obtained using the $L_{1}$ and $L_{\infty}$ distances on the same point sites.

While these diagrams are not printable - due to the horizontal facets - an interesting emerging property is that the facets take only a fixed number of different angles. This, in fact, is a property of any Voronoi diagram under a polyhedral distance: a distance induced by a convex polyhedron [Icking and Ha 2001; Ma 2000].

Our intuition is that some specific convex polyhedron must exist that induces polyhedral Voronoi diagrams whose facet set is fully fabricable. Figure 2 (rightmost) shows an initial test that confirmed this intuition: using a cone distance produces a fully printable diagram. This idea forms the basis of our approach.

We detail how we build upon this initial intuition to define parameterized 3D Voronoi diagrams in Section 4. We analyze the mechanical behavior of the structures in Section 5 and demonstrate applications to additive manufacturing in Section 6.

\section{METHOD}

We now describe the use of polyhedral Voronoi diagrams for producing microstructures with spatially varying elasticity, well suited for continuous deposition (FFF-like processes).

Let $B_{d}=\left\{p: p_{z}=d\right\}$ be a plane orthogonal to the printing direction $z$, and $B_{d}^{-}=\left\{p: p_{z}<d\right\}$ be a half-space below $B_{d}$. We call slope of a facet or slope of a plane the dihedral angle between the plane supporting the facet and any horizontal $(x y)$ plane. For example a vertical wall has slope $\pi / 2$, the printing plane slope zero.

Our microstructure is defined by a mesh $\mathcal{K}$ that must satisfy the following two fabrication constraints:

(1) Angle constraint: Each facet $f$ of $\mathcal{K}$ has to be "sufficiently close to vertical" so that it is printable with FFF. We model this constraint by imposing a lower-bound $\theta^{\star} \in[0, \pi / 2]$ on the slope of facet $f$. If $n$ is the normal vector of facet $f$, it must satisfy $\left|n_{z}\right| \leq \cos \theta^{\star}$. Reasonable values of $\theta^{\star}$ may range, say, from $\pi / 6$ to $\pi / 3$ and depends on the fabrication hardware at hand.

(2) No-local-minimum constraint: Each point $p \in \mathcal{K}$ has to be "supported from below" so that it does not appear mid-air during fabrication; i.e. we have that $\mathcal{K} \cap N(p) \cap B_{p_{z}}^{-} \neq \emptyset$ where $N(p)$ is any open neighborhood of $p$. 
These two constraints are well-suited for FFF-like processes, as they capture the requirement that deposited material has to be well supported from below. For our purpose in this paper, we model the mesh $\mathcal{K}$ as the union of the facets of a polyhedral Voronoi diagram, namely, the Voronoi diagram of a set of point sites that arises when using a specific polyhedral distance instead of the usual Euclidean one (Section 4.1.1). We show that some choices of distance polytopes guarantee that $\mathcal{K}$ satisfies both fabrication constraints. In particular, we show how one can easily check that a given polyhedral distance entails a fabricable mesh $\mathcal{K}$ (Section 4.1.4) and we exhibit a simple family of such polytopes (cones, Section 4.2). We discuss which parameters can be spatially graded and how (Section 4.3). Finally, in Section 4.4 we detail a simple algorithm to extract the deposition paths delineating to the Voronoi facets.

\subsection{Polyhedral Voronoi diagrams}

We now recall the definition of polyhedral distances, polyhedral bisectors, and their use in Voronoi diagrams. For simplicity, some of the following illustrations consider a planar polygonal distance.

4.1.1 Polyhedral distances. Let $P \subset \mathbb{R}^{3}$ be a compact convex polyhedron of combinatorial size $k$ containing the origin in its interior. The polyhedral distance induced by $P$ from a point $p$ to $q$ is (e.g., [Okabe et al. 2009]):

$$
d_{P}(p, q)=\min \{t \geq 0: q \in p+t P\} .
$$

The value of $d_{P}(p, q)$ is the minimal scaling factor $t$ applied to $P$ so that $p+t P$ touches $q$ (Figure 3 ). Note that the $L_{1}$ and $L_{\infty}$ distances are particular cases of polyhedral distances. Since $P$ is convex, the triangle inequality holds: $0 \leq d_{P}(p, r) \leq d_{P}(p, q)+d_{P}(q, r)$ (proof in supplemental material). However, $d_{P}$ is symmetric if and only if $P$ is centrally symmetric with respect to the origin.

The distance $d_{P}(p, q)$ can be computed as follows [Icking et al 1995]. Let $q^{\prime}$ be the unique point of $p+P$ intersected by the ray from $p$ to $q$ (see Figure 3). Then $d_{P}(p, q)=\frac{\|q-p\|}{\left\|q^{\prime}-p\right\|}$.

In our implementation, we compute the closest intersection of the ray with the planes supporting the facets of $P$.

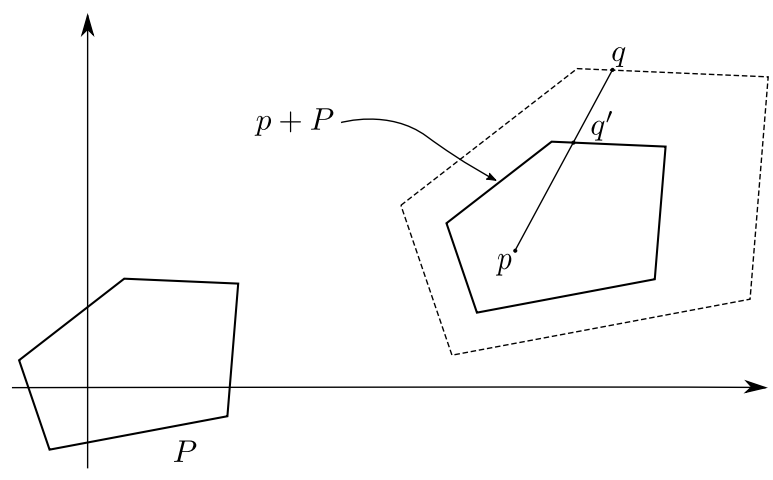

Fig. 3. A polygonal distance function.
4.1.2 Polyhedral bisectors. The bisector between two point sites $p$ and $q$ is the set of points equidistant to $p$ and $q$ under the distance $d_{P}$, that is:

$$
\mathcal{B}(p, q)=\left\{r \in \mathbb{R}^{3}: d_{P}(p, r)=d_{P}(q, r)\right\}
$$

Note that the polyhedral distance is taken from $p$ and $q$ to $r$ because $d_{P}$ is not symmetric.

4.1.3 Polyhedral Voronoi diagrams. Equipped with the polyhedral distance, we now describe their use in Voronoi diagrams. Let $S$ be a set of $n$ point sites in $\mathbb{R}^{3}$. The polyhedral Voronoi diagram $\operatorname{Vor}_{P}(S)$ is the decomposition of $\mathbb{R}^{3}$ into Voronoi cells. A cell $V_{P}\left(s_{i}\right)$ for a site $s_{i} \in S$ is defined as:

$$
V_{P}\left(s_{i}\right)=\left\{p \in \mathbb{R}^{3}: d_{P}\left(s_{i}, p\right) \prec d_{P}\left(s_{j}, p\right), \forall s_{i} \neq s_{j} \in S\right\}
$$

where the lexicographical distance relation $\prec$ is necessary to deal with cases where a full region space belongs to the bisector (nongeneral position cases [Klein and Wood 1988]), see Figure 4:

$$
\begin{gathered}
d_{P}\left(s_{i}, p\right)<d_{P}\left(s_{j}, p\right) \Longleftrightarrow \\
d_{P}\left(s_{i}, p\right)<d_{P}\left(s_{j}, p\right) \vee\left(d_{P}\left(s_{i}, p\right)=d_{P}\left(s_{j}, p\right) \wedge(i<j)\right)
\end{gathered}
$$

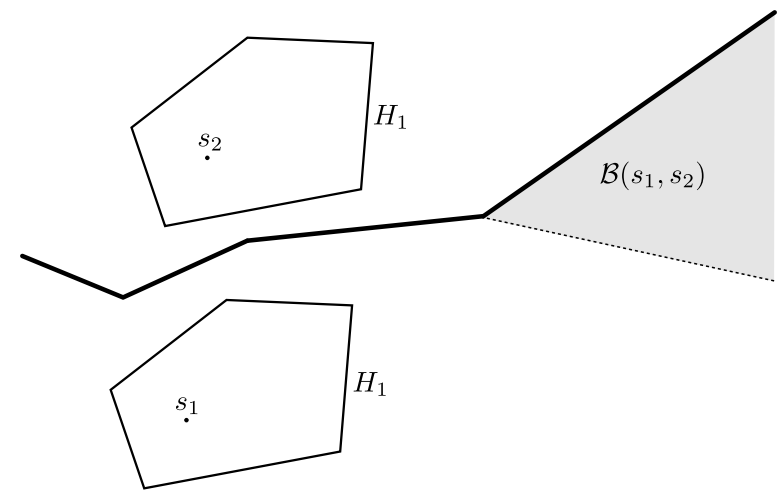

Fig. 4. A non-general position case: the line passing through $s_{1} s_{2}$ is parallel to the line containing the facet $H_{1}$ of $P$. The gray region are points of the polyhedral bisector $\mathcal{B}\left(s_{1}, s_{2}\right)$. The lexicographical distance of Equation (5) selects one of the boundaries of the gray region.

The Voronoi facets form the boundary set of Voronoi cells. Due to the triangle inequality, the Voronoi cells are star-shaped polyhedrons [Chew and Dyrsdale 1985]: for all $q \in V_{P}(s)$ the line segment from $s$ to $q$ is in $V_{P}(s)$. Regarding the combinatorial complexity, assuming the complexity $k$ of $P$ is constant, the worst case complexity of the Voronoi diagram is $\Theta\left(n^{2}\right)$ [Icking and Ha 2001] $(n=|S|)$.

An important property for our purpose is that the facets of $\operatorname{Vor}_{P}(S)$ form a subset of the arrangement of the bisectors of each pair of sites in $S \times S$ (see, e.g., [Ma 2000]). Thus, if all the bisectors satisfy both fabricability constraints then the facets of the Voronoi diagram form a fabricable mesh as well. We now analyse polyhedral bisectors in more detail. 
4.1.4 Properties of polyhedral bisectors. In this section, we recall known facts about polyhedral bisectors and explain how these properties can be used to check whether the geometry of $P$ induces fabricable bisectors. Various works have already studied polyhedral bisectors [Corbalan et al. 1996; Icking et al. 2001; Martini and Swanepoel 2004]. For a more detailed exposition, we refer the reader to the thesis of Ma [2000] and the references therein.

For simplicity, we assume that two points $p$ and $q$ are in general position with respect to $P$. That is, the line through $p$ and $q$ is not parallel to any line segment contained in the boundary of $P$ [Boissonnat et al. 1995]. Then, $\mathcal{B}(p, q)$ is piecewise linear and homeomorphic to a plane [Ma 2000]. We give a method to construct the bisector of two point sites in the supplemental material.

We define a feature as either a vertex, a (straight) edge or a (planar) facet. We say that two features $X \subset P$ and $Y \subset P$ on the boundary of the distance polytope $P$ generate a feature $B$ of the bisector $\mathcal{B}(p, q)$ when $B=\bigcup_{\lambda>0}((p+\lambda X) \cap(q+\lambda Y))$. $B$ is either empty or does not depend on the relative position of the point sites $p$ and $q$. It follows from the constructions of polyhedral bisector (detailed in the supplemental material) and Proposition 4.1 below, that each feature of a bisector is generated by two features on $P$.

To determine fabricability, we have to analyse the slope of the bisector facets. This builds upon the following property. Let $B_{i j}$ be a facet of the bisector $\mathcal{B}(p, q)$ generated by the facets $f_{i}$ and $f_{j}$ of $P$. Let $n_{i}$ be the outward normal vector to facet $f_{i}$. Let $\alpha_{i}>0$ be the distance from the origin to the plane $H_{i}$ supporting $f_{i}$. (In particular, if $r$ is a point of $f_{i}$ then $\alpha_{i}=r \cdot n_{i}$.)

Proposition 4.1. Write $H_{i j}$ for the plane that spans the origin and the line $H_{i} \cap H_{j}$. Then, the bisector facet $B_{i j}$ (generated by $f_{i}$ and $f_{j}$ ) is parallel to the plane $H_{i j}$ (Figure 5). The normal vector of $B_{i j}$ is proportional to $\alpha_{j} n_{i}-\alpha_{i} n_{j}$.

The proof is in [Icking and Ha 2001]. The derivation of the formula for the normal vector can be found in the supplemental material. Given Proposition 4.1, we now explain how to check if both fabrication constraints are respected.

Verifying the angle constraint. Each planar facet $B_{i j}$ of the bisector $\mathcal{B}(p, q)$ is defined through the interaction between two facets $f_{i}$ and $f_{j}$ of $P$ (see Figure 5). Proposition 4.1 tells us that the normal vector of $B_{i j}$ depends only on $f_{i}$ and $f_{j}$ and is independent of the relative position of $p$ and $q$. Thus, there are at most $\left(\begin{array}{l}k \\ 2\end{array}\right)$ different orientations for the bisector facets. We can check that they satisfy the angle constraint by enumerating all pairs of facets of $P$.

Verifying the no-local-minimum constraint. Similarly, the vertices of all bisectors exhibit a finite number of configurations that can be enumerated in time $O\left(k^{2}\right)$, to verify that no vertex of any bisector is a local minimum with respect to the build direction. Each bisector vertex is generated by either an edge-edge pair or a vertex-facet pair (see Figure 6). Details are given in the supplemental material.

In summary, for a given polyhedral distance $d_{P}$ induced by a polytope $P$ of size $k$, we can check in time $O\left(k^{2}\right)$ if the polyhedral bisectors under $d_{P}$ satisfy both fabrication constraints.

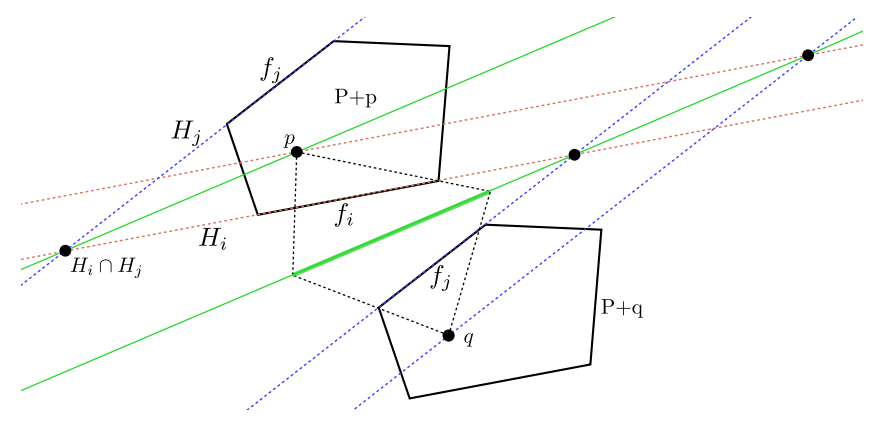

Fig. 5. Let $f_{i}, f_{j}$ be facet of $P$. Let $H_{i}$ and $H_{j}$ be the supporting planes of $f_{i}$ and $f_{j}$, respectively. The feature of $\mathcal{B}(p, q)$ generated by $f_{i}$ and $f_{j}$ is drawn bold and green. A construction for its supporting plane is shown. Lines with the same color are parallels. The bold green bisector feature is indeed parallel to the top green line, as constructed in Proposition 4.1. See Lemma 3.2.1.2 in [Ma 2000].

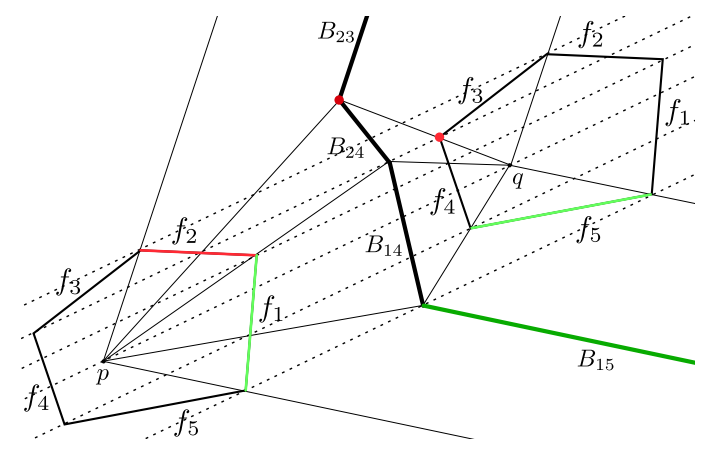

Fig. 6. Polygonal bisector between two point sites $p$ and $q$. Each facet $B_{i j}$ of the bisector is generated by facets $f_{i}$ on $p+P$ and $f_{j}$ on $q+P$. The dark-red bisector vertex is generated by the two light-red features of $P$. The dark-green bisector edge is generated by the two light-green features of $P$. A section in the supplemental material details this geometric construction.

\subsection{Polyhedral cone distances}

We now describe our choice of a particular family of polyhedral distances that induce fabricable Voronoi diagrams. The ideal family has few parameters and the influence of each parameter should be well understood, and easy to relate to the final elastic behavior. We prefer a distance that is symmetric around the $z$-axis so that it is easier to reason about. The most difficult property to achieve is the absence of local minimum.

We determined that a polyhedral cone achieves these objectives, and we now describe our family of cone distances. Given the parameterized cone geometry (Section 4.2.1), we explain how the fabrication constraints are satisfied (Section 4.2.2) and extend the family with anisotropic cones (Section 4.2.3). We highlight the influence of the parameters on the induced Voronoi diagram in Section 4.3.

4.2.1 Geometry of the cone. The base facet of the cone is a horizontal regular $k$-gon (Figure 7-bottom-left). The cone has $k$ side facets (typically $k=8$ ). The scale of the distance polytope does not affect the Voronoi diagram that it induces, so we assume that the base facet lies at $z=-1$. The cone has apex $A=\left(0,0, A_{z}\right)$ 


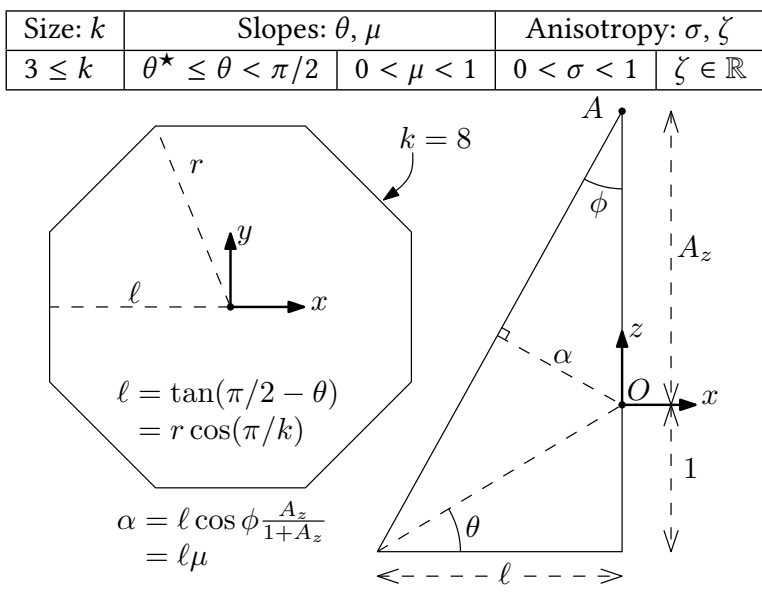

Fig. 7. Top. The parameters describing our family of distances. Bottom. The geometry of the cone for $k=8$. The right triangle is the half of the cone cutaway in the plane $(O, x, z)$. We indicate remarkable quantities used in computations.

with $A_{z}>0$. (Figure 7-right). The vertices of the base facet have $y,-x$-coordinates: $r \cos a_{i}$ and $r \sin a_{i}$ where $a_{i}=(2 i+1) \frac{\pi}{k}$ for $i=0 . . k-1$, and $r$ is the radius.

We parameterize the cone with parameters $k, \theta, \mu$ (Figure 7-top) and add parameters $\sigma, \zeta$ for controlling anisotropy (Section 4.2.3). All other quantities are determined from these parameters to enforce fabrication constraints (Section 4.2.2). Examples of cones within our family are shown in Figure 8.

4.2.2 Cones for fabricable Voronoi diagrams. We now consider constraints on the slopes of the bisector features. All the side facets contain the apex $A$ of the cone, thus if $H_{i}$ and $H_{j}$ are planes supporting two side facets $f_{i}$ and $f_{j}$, their intersection line contains $A$ as well. By Proposition 4.1, $H_{i j}$ contains both $A$ and the origin $O$, so that it is a vertical plane. We conclude that any bisector feature generated by two side facets is vertical and therefore satisfies the angle constraint.

Let us now consider a feature generated by a side facet and the base facet. By Proposition 4.1, this feature is parallel to a plane spanning an edge of the base facet and the origin. Its slope $\theta$ (Figure 7-bottom) is the same for all side facets. In order to satisfy the angle constraint, we choose the value of $\theta$ in the range $\left[\theta^{\star}, \pi / 2\right.$ ), where $\theta^{\star}$ is the angle constraint bound. This fixes the value of $\ell=\tan (\pi / 2-\theta)$ and the radius $r=\ell / \cos (\pi / k)$ of the base facet.

The last value to determine is $A_{z}$. It comes from our parameter $\mu$, which controls the distance $\alpha$ from the origin to a side facet. By expressing the sine of angle $\phi$, we compute $\alpha=\ell \cos \phi \frac{A_{z}}{1+A_{z}}$. In order to decouple parameters $\theta$ and $\mu$, we interpret our parameter $\mu$ as $\mu=\alpha / \ell$. Then, by expressing the squared tangent of angle $\phi$ as $\frac{\alpha^{2}}{A_{z}^{2}-\alpha^{2}}=\frac{\ell^{2}}{\left(1+A_{z}\right)^{2}}$, we obtain $A_{z}=\frac{\mu}{1-\mu^{2}}\left(\mu+\sqrt{1+\ell^{2}\left(1-\mu^{2}\right)}\right)$. Figure 9 shows a $2 \mathrm{D}$ example cone (a triangle). The table of possible bisector vertices shows the impossibility of a local minimum. We provide in the supplementary material an interactive applet illustrating the induced bisectors in 3D.
4.2.3 Anisotropic cones. We vary the anisotropy of the polyhedral Voronoi diagram by shrinking the distance cone along the $x$ axis followed by rotating it around the $z$ axis. We do not allow other axes of rotation so as to guarantee that the Voronoi mesh stays fabricable: the base of the cone becomes a polygonal approximation of an ellipse and stays horizontal. The shrink is obtained by applying to the cone the linear transformation $\operatorname{diag}(\sigma, 1,1)$ where $0<\sigma<1 .{ }^{3}$ The rotation angle is specified with parameter $\zeta$. The analysis in Section 4.2.2 still applies. A pair of side facets generate a vertical bisector feature and the base facet generates features with slopes that vary for each side facet, but are always larger than or equal to $\theta$. Figure 8 illustrates how the parameterization impacts the corresponding polyhedral Voronoi facets.

\subsection{Spatial variations}

The capacity to obtain gradients of properties within fabricated shapes is a major advantage offered by additive manufacturing. In this section we discuss how the geometric properties of our microstructures can vary spatially.

4.3.1 Varying the point sites density. The microstructures that we fabricate are materializations of the Voronoi mesh $\mathcal{K}$. We control their density by changing the set of point sites $S$ on which the Voronoi diagram is computed, following an input density field $\mathbb{R}^{3} \rightarrow \mathcal{D}$ where $\mathcal{D} \subset \mathbb{R}$ is a range of fabricable densities. In practice we follow the implementation of Martínez et al. [2016] to produce point sites in a multi-resolution jittered grid.

4.3.2 Varying the polyhedral distance. We now consider that each of the parameters $\theta, \mu, \sigma$ and $\zeta$ are fields in $\mathbb{R}^{3}$. The value of the parameter is picked at the location of a point site $s \in S$, and used to define the distance polyhedron at $s$. Thus, we are effectively using a different distance for each site in $S$. We discuss two subsets of parameters that can be spatially varied together while still guaranteeing fabricability in Section 4.3.3 and Section 4.3.4. We always consider $k \geq 3$ and $\theta \geq \theta^{\star}$ constant.

Remark. When changing the distance at each site, the fact that the bisector between two sites $p$ and $q$ is homeomorphic to a plane does not hold anymore. In fact, in such a situation, the bisector can have more that one connected component [Icking et al. 2001]. We never observed this behavior in our experiments. This is due to the fact that point sites are distributed all over space so that pieces of bisectors that may appear far from their two parent sites are "taken over" by the Voronoi cells of other sites.

4.3.3 Varying $\mu$. The apex of the cone moves vertically from just above the origin when $\mu \approx 0$ to infinitely high as $\mu$ approaches 1. Consider two side facets of two identical cones and start raising the apex of one cone (but not the other). Then, the feature that those facets generate becomes increasingly horizontal. The slope decreases but fortunately never reaches below $\theta$.

While we do not have a formal proof, we can verify it numerically. Consider a side facet $f_{i}$ of a cone with a value $\mu<1$ together with each side facet $f_{j}$ of a cone with $\mu=1$, i.e., $f_{j}$ is vertical. Each pair

\footnotetext{
${ }^{3}$ Let $\eta$ be the minimum slope of the bisector facets. In order to have $\eta$ stay constant when we shrink the cone along the $x$ axis, we generate the circle in the $y,-x$ frame so that the cone has at least one side facet perfectly parallel to the $x$ axis.
} 

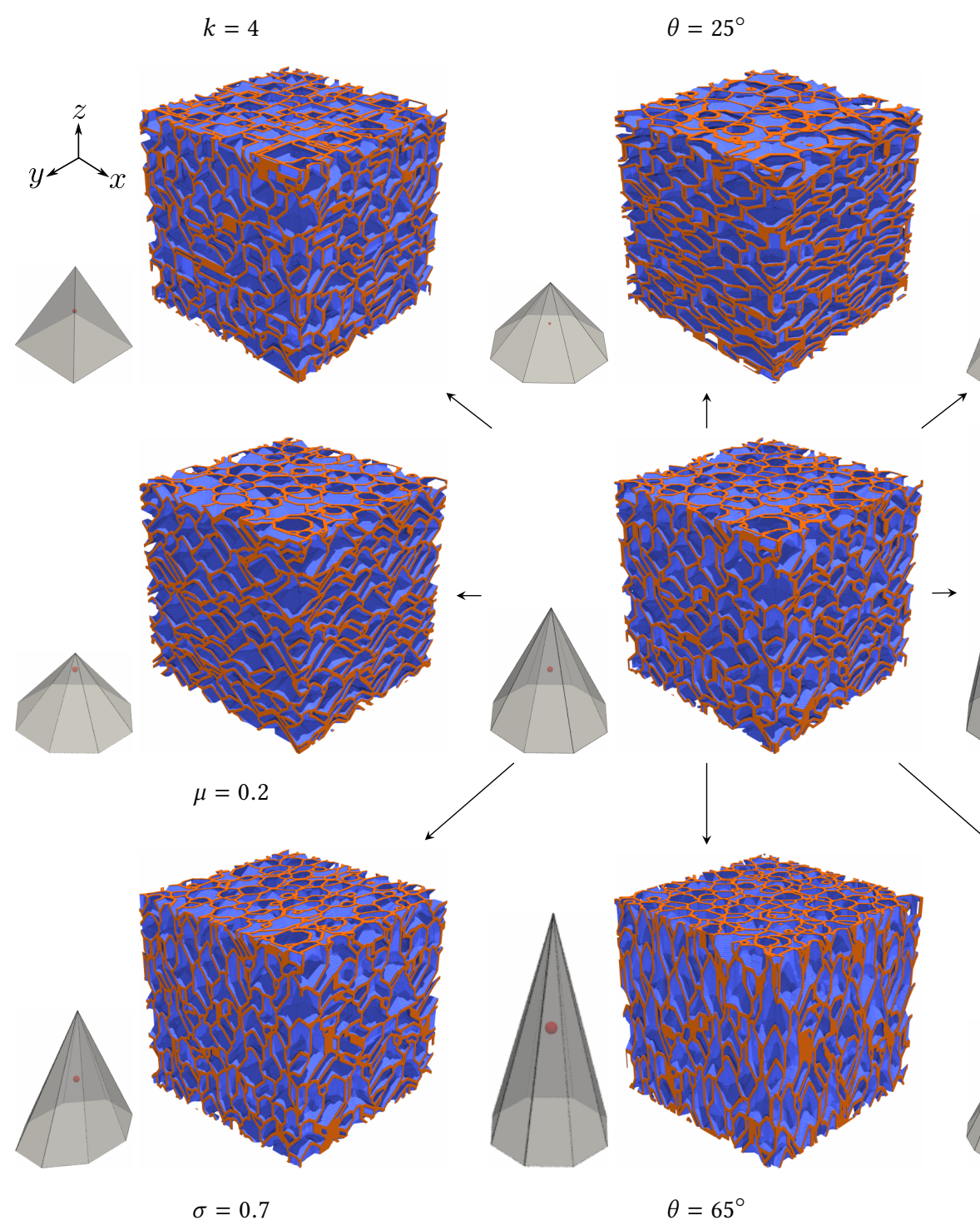

$$
\sigma=0.4
$$




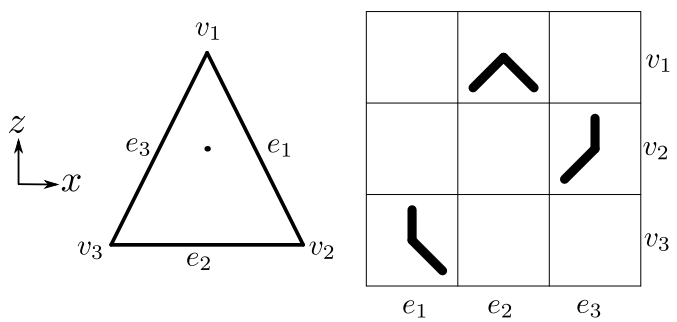

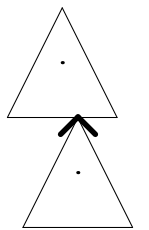

$v_{1} e_{2}$

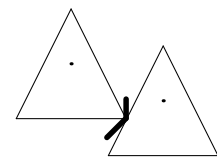

$v_{2} e_{3}$

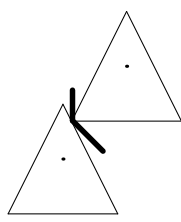

$v_{3} e_{1}$
Fig. 9. Polygonal distance defined by a triangle. The three different bisector vertex configurations are shown in the table, and in context below. The polygonal bisectors do not exhibit any local minimum, and form an angle of at least $45^{\circ}$ with respect to the $x$ axis.

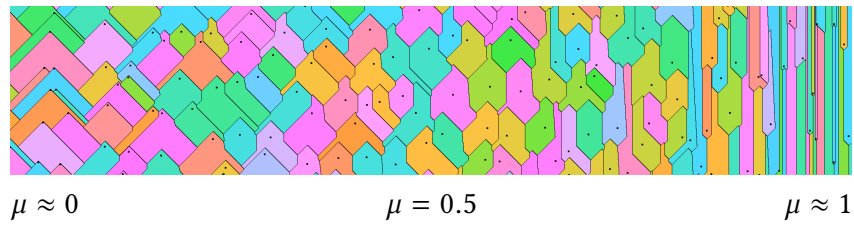

Fig. 10. Varying parameter $\mu$ with $\theta=45^{\circ}$.

Changes in the Voronoi mesh. Geometrically, as $\sigma$ decreases, the Voronoi cells become flatter in a direction specified by $\zeta$. This flattening also increases the slope of some bisectors (as we have seen in Section 4.2.3), especially those parallel to the anisotropy direction. Compare, for example, Figure 8 center and top-right.

4.3.5 Varying $\sigma, \zeta, \mu$ and $\theta$. It is possible to vary all four parameters simultaneously. In that case however, $\mu$ and $\theta$ are interdependent, and function as a single parameter. The supplemental material gives more details. We have not experimented with this 3-parameters family yet, and set it as future work.

\subsection{Algorithm and implementation}

For fabrication we seek to efficiently generate single slices of the microstructures. In order to print with the smallest available thickness, we produce paths delineating the Voronoi facets - these paths have no thickness, since the thickening comes from the physical material deposition. To guarantee scalability, we devise a procedural approach relying on discretization, as illustrated in Figure 11. We overlay a 2D regular grid over the slice, called the labels grid and label each grid square with the site of $S$ closest to the square center, as measured by the polyhedral distance. Then, we extract all the boundary edges between adjacent grid squares with different labels and simplify the extracted jagged paths in order to produce the final deposition paths for fabricating the slice of microstructure.
This affords for a simple space-tiling approach, limiting required memory and enabling parallelism.

There are many interesting details on how to implement this efficiently, and we refer the interested reader to the supplemental material. Our implementation uses OpenCL for parallel grid labeling, and $\mathrm{C}++$ for path extraction. On a GeForce GTX 1080 and an Intel $\mathrm{i} 7-6800 \mathrm{~K}$, the Voronoi labeling for $1000^{2}$ labels takes around $20 \mathrm{~ms}$ and the path extraction $1600 \mathrm{~ms}$ (the cone parameters are $\left.\theta=45^{\circ}, k=8, \mu=0.6, \sigma=1\right)$. The current bottleneck lies in the path extraction since we use an unoptimized sequential method.

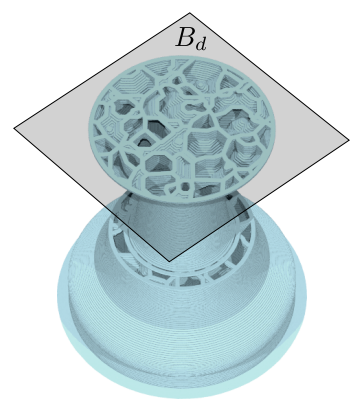

(a) Current intersection.

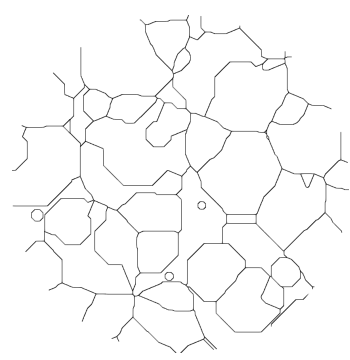

(c) Boundary extraction.

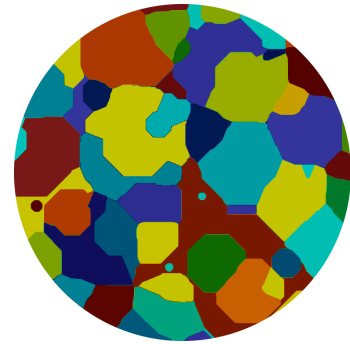

(b) Voronoi labeling.

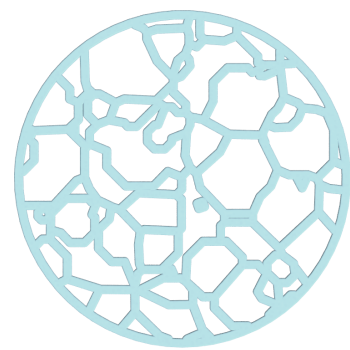

(d) Path generation.
Fig. 11. Overview of the path extraction. The input solid $C$ is a bishop chess piece (https://www.thingiverse.com/thing:378322/). (a) The bishop cut by the plane $B_{d}$. (b) We label each square in the labels grid with its closest site in $S$. Each Voronoi cell is shown with a different color. (c) We extract the axis-aligned edges between Voronoi cells. (d) We generate simplified deposition paths inside $C$.

\section{ELASTIC BEHAVIOR}

We analyze the elasticity of polyhedral Voronoi diagrams with numerical periodic homogenization akin to [Martínez et al. 2016; Panetta et al. 2015; Schumacher et al. 2015]. Periodic homogenization seeks to find the elasticity tensor characterizing a periodic composite material defined from a unit periodic cell. We do not reproduce here all the details and refer the interested reader to the aforementioned publications and our supplemental material.

One difference with prior works is that we use the CrAFT software [Boittin et al. 2014], based on the fast Fourier transform (FFT) [Moulinec and Suquet 1998]. In comparison to finite element methods, it allows us to homogenize high resolution volumes, achieving higher precision. CrAFT has been successfully used to homogenize porous microstructures such as Voronoi foams [Boittin et al. 2017]. 
After analysis, we consider the parameters of the fitted orthotropic material, primarily the Young's moduli $E_{x}, E_{y}, E_{z}$ along respectively the $\mathrm{x}, \mathrm{y}$ and $\mathrm{z}$ axis (measure of stiffness). We provide analysis of the shear moduli $G$ and Poisson's ratio $v$ in supplemental material, as well as the homogenized tensors obtained by this procedure.

We are also interested in evaluating the overall compressive strength. To do so, we consider the bulk modulus $K$, that characterizes the tendency of a material to deform in all directions when uniformly loaded in all directions. A high bulk modulus implies higher incompressibility. We consider the Voigt-Reuss average $K_{V R}$ of the bulk modulus bounds [Hill 1952] (details in supplemental material).

All analysis is performed with a base isotropic material having Young's modulus $E=1$ (normalized) and Poisson's ratio $v=0.3$. Thus, it is expected that $E_{i} \in[0,1]$. The Poisson's ratio of an orthotropic material is unbounded [Ting and Chen 2005]. The CrAFT convergence threshold is set such that the modulus of the divergence of the stress field is lower than 0.005 , providing convergence and a reasonable trade-off between accuracy and performance.

\subsection{Material space exploration}

We explore the material space spanned by the parameterized cone distance (Section 4.2). For all tests the unit periodic cell is discretized into $200^{3}$ voxels and the thickness of Voronoi facets is 0.03 .

We first consider a point density $\rho$ of 125 point sites per unit cell (given by a $5 \times 5 \times 5$ jittered grid point distribution). We evaluate the Cartesian product of the following cone parameters, for 5 different random realizations of point sites, performing in total 2205 tests:

- $\theta \in\left\{25^{\circ}, 45^{\circ}, 65^{\circ}\right\}$

- $k \in\{4,8,16\}$

- $\mu \in\{0.2,0.3,0.4,0.5,0.6,0.7,0.8\}$

- $\sigma \in\{1.0,0.9,0.8,0.7,0.6,0.5,0.4\}$

The material space we explored spans a range of relative volumes $\mathcal{V} \in[0.413,0.634]$ for unit cells. The results for the Young's moduli are shown in Figure 12, offering a wide range of possibilities along each orthotropy axis. The results for Poisson's ratio and shear moduli are shown in the supplemental material, spanning a variable range of elastic behaviors, with an always positive Poisson's ratio.

We observe a good correlation between the cone parameters and the orthotropic elastic constants, as detailed in Section 5.2. The variance of the logarithmic Euclidean distance [Moakher and Norris 2006] between each 5 random realizations of points sites having the same parameters remains low at 0.0006 , indicating that randomness has a limited impact. In addition, we verified that zero entries of the ideal elasticity orthotropic tensor differ by at most 0.005 from the homogenized one, indicating that an orthotropic material constitutes a plausible approximation.

\subsection{Influence of parameters}

We now consider how each parameter of the polyhedral distance impacts the mechanical response.

Influence of $\theta$. Increasing $\theta$ increases further $E_{z}$, since the Voronoi facets become closer to being vertical (see Figure 8).
Influence of $\mu$. Increasing $\mu$ mainly increases $E_{z}$, since the area of vertical Voronoi facets increases (see Figure 8). In addition, increasing $\mu$ mainly decreases $v_{y z}$, increases $v_{z x}$, and decreases $G_{y z}, G_{z x}$ in a different degree depending on $\theta$ (see supplemental material).

Influence of $\sigma$. Decreasing $\sigma$ decreases $E_{x}$ and increases $E_{y}, E_{z}$, since the Voronoi facets become comparatively more elongated in the $y$ and $z$ direction than in the $x$ direction (see Figure 8). In addition, decreasing $\sigma$ mainly increases $v_{z x}$, decreases $v_{x y}$, and increases $G_{x y}$ (see supplemental material).

Influence of $k$. We observe that the value of $k$ impacts on the following two properties:

- Increasing $k$ decreases the variance of the homogenized elasticity under different random distributions of the point sites. For each 5 random realizations with same parameters, the average logarithmic Euclidean distance between any pair of tensors is $11 \%$ lower for $k=8$ (similar for $k=16$ ) with respect to $k=4$.

- Increasing $k$ increases the Voigt-Reuss average bulk modulus. In particular, for $k=4$ the average unit cell volume is $\mathcal{V}=$ 0.481 and $K_{V R}=0.107$, for $k=8$ we have $\mathcal{V}=0.469$ and $K_{V R}=0.11$. Thus, $k=8$ exhibits a higher average $K_{V R}$ (similar for $k=16$ ) while having an even lower $\mathcal{V}$.

Thus, a value of $k \geq 8$ is encouraged to achieve better strength to weight ratio, and being closer to the homogenized analysis.

Influence of point density. Increasing the point density $\rho$ increases the overall elastic moduli, since the unit cell volume $\mathcal{V}$ increases [Gibson and Ashby 1999]. This is illustrated in Figure 13, where we evaluate additional point densities $\rho$.

\subsection{Material space control}

An orthotropic material is defined by nine independent constants (see supplemental material). For a fixed $\theta, k$, and $\zeta$, we have three independent parameters $(\rho, \mu, \sigma)$ controlling the microstructure geometry. Thus, our microstructure only spans a subset of the space of orthotropic materials.

In the context of material design, one usually seeks to independently control the three orthogonal Young's moduli $E_{x}, E_{y}, E_{z}$. In order to unveil the correlation of the different microstructure parameters and the other elastic constants we propose to visualize each as a function of $\left(E_{x}, E_{y}, E_{z}\right)$. Results are reported in Figure 14.

To provide a dense visualization from the discrete sampling of the material space, we interpolate the space of variables (see Section 5.1). We seek to approximate a function $f\left(E_{x}, E_{y}, E_{z}\right)=(\rho, \mu, \sigma)$. We perform a multivariate interpolation of the function $f$ implemented by the Python package scipy.interpolate. Other triplets of physical parameters could be chosen to perform this analysis. However, the space of Young's moduli provides the largest design freedom.

Design freedom. From the designer perspective the material space offers the following controls. The Young's moduli may be freely changed along each axis of the orthotropic frame. The $z$ axis of the orthotropic frame has to remain aligned with the build direction. The other axes may otherwise be freely rotated (parameter $\zeta$ ). 

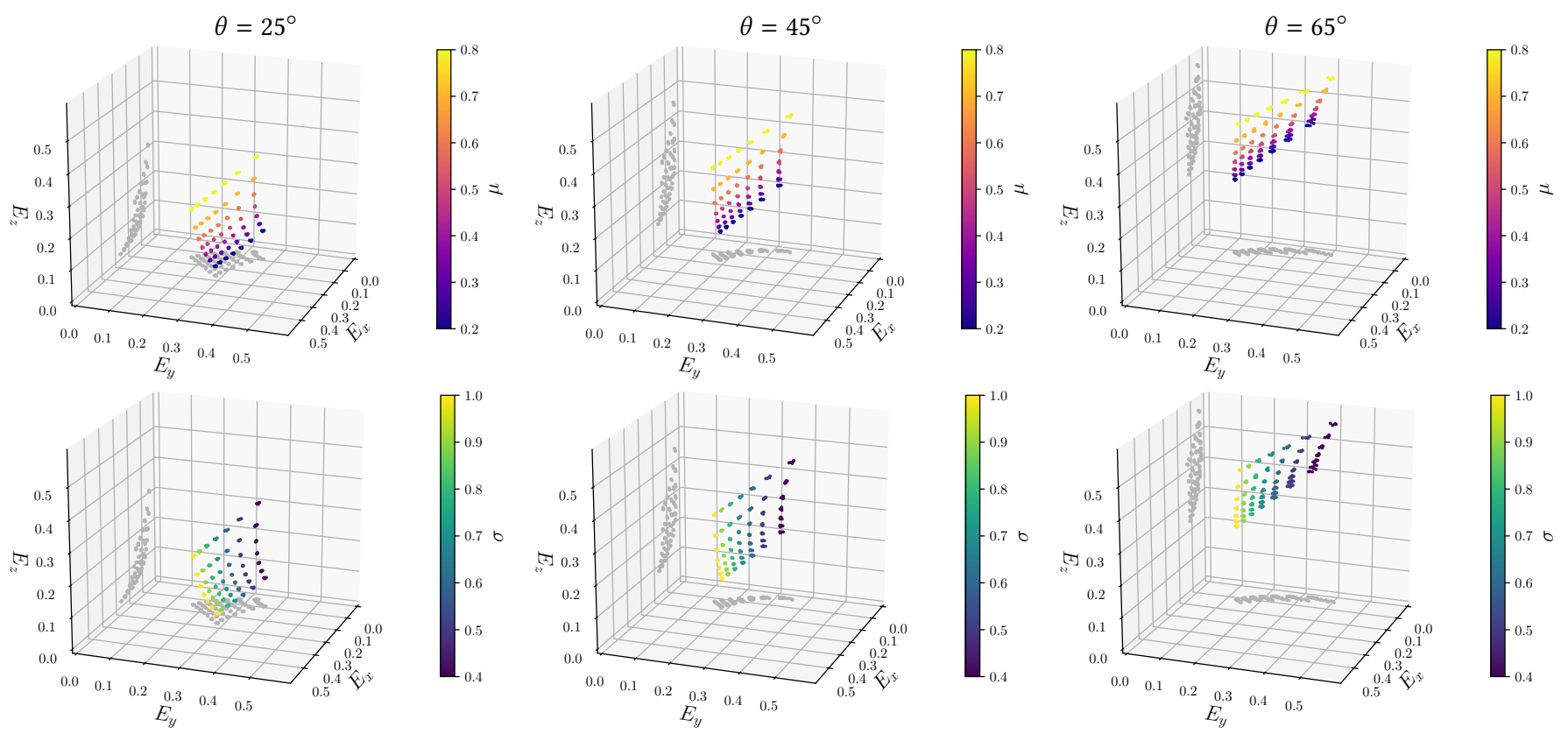

Fig. 12. Material space for $k=8$ and $\rho=125$. Each column corresponds to a different minimal bisector slope $\theta$. Each plot dot denotes a single test. The plot axes correspond the three orthogonal Young's moduli. The color of dots either depicts $\mu$ (first row) or $\sigma$ (second row). For better interpretation, each dot is projected in gray color to the $E_{z}$ and $E_{y}$ planes.

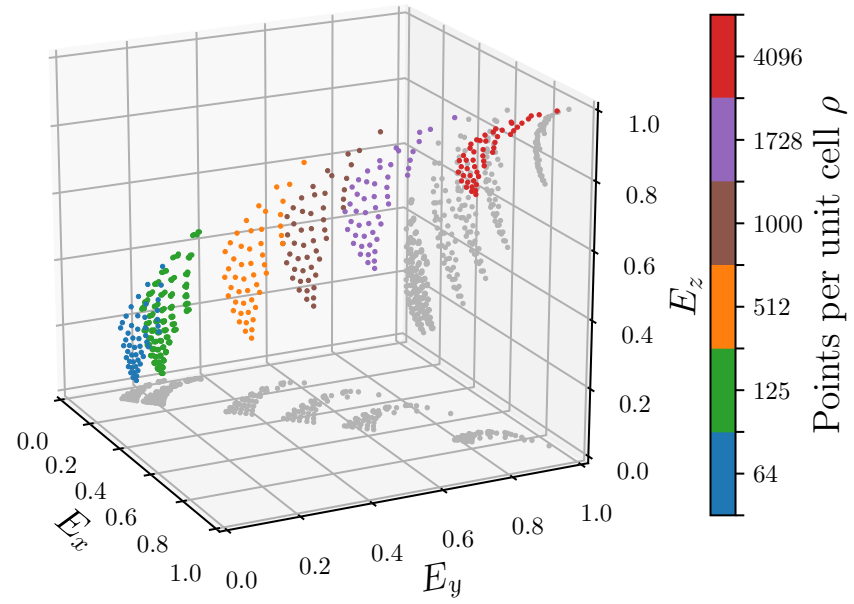

Fig. 13. Material space for $k=8$ and $\theta=45^{\circ}$. We consider the same range of parameters $\sigma$ and $\mu$ used in Figure 12, while varying in addition the point density for $\{64,125,512,1728,4096\}$ point sites per unit cell. The data for 125 point sites per unit cell (green points) corresponds exactly to $\theta=45^{\circ}$ in Figure 12 (middle row). For better interpretation, each dot is projected in gray color to the $E_{z}$ and $E_{y}$ planes.

These variations may be spatially graded. Currently we limit gradations to either $\mu$ (Section 4.3.3) or $\sigma$ and $\zeta$ (Section 4.3.4), but full variations are possible (Section 4.3.5). We show in Figure 15 the effect of fixing either $\mu$ or $\sigma$ and varying the other. Fixing one spans a subset curve within $\left(E_{x}, E_{y}, E_{z}\right)$.

\subsection{Experimental verification}

We performed experimental verification of the predicted elastic behavior. We print three samples with varying orthotropy and measure their linear elastic response (Young's modulus) using a compression test along each axis. The results are reported in Figure 17. To mitigate the impact of the choice of printer, we use three different machines (see Figure for details).

The experimental results are consistent with numerical results, even though there are differences. The main one is a lower stiffness along the build direction, on average. This is to be expected since layered materials are anisotropic. As future work, we would like to investigate homogenization with an anisotropic material for AM [Liu and Shapiro 2016]. Nevertheless, the discrepancies are limited and we observe a direct correlation between control parameters $(\mu, \sigma)$ and the measured Young's moduli. We provide curves for one sample in Figure 18, revealing the non-linear behavior under large compressions. The curve reveals a smooth response with the expected flattening. We also performed a repeatability test, taking five measurements along each axis of a same sample. We observe variations of $\pm 3 \%$ without any noticeable trend.

\subsection{Comparison to tessellations by planes}

Self-supported structures made of interleaved sets of parallel planes have become a popular choice for filling volumes in the context of FFF [Lee and Lee 2017; Lefebvre 2015; Wu et al. 2016]. ${ }^{4}$ To the best of our knowledge, the material space spawned by these infills has

\footnotetext{
${ }^{4}$ See also cubic/tetrahedral infill in software Cura.
} 



Fig. 14. Interpolation given by the material space sampling shown in Figure $13\left(\theta=45^{\circ}, k=8\right)$. The plot axes correspond the three orthogonal Young's moduli. Both the Poisson's ratio $v_{x y}, v_{z x}, v_{y z}$ (always positive), and the shear moduli $G_{y z}, G_{z x}, G_{x y}\left(G_{\max }=\frac{E}{2(1+v)} \approx 0.38\right.$ for base material $E=1, v=0.3$ ) remain fixed for a desired triplet of Young's moduli. The plots unveil the good correlation between the parameters $\rho, \mu, \sigma$ and the Young's moduli.
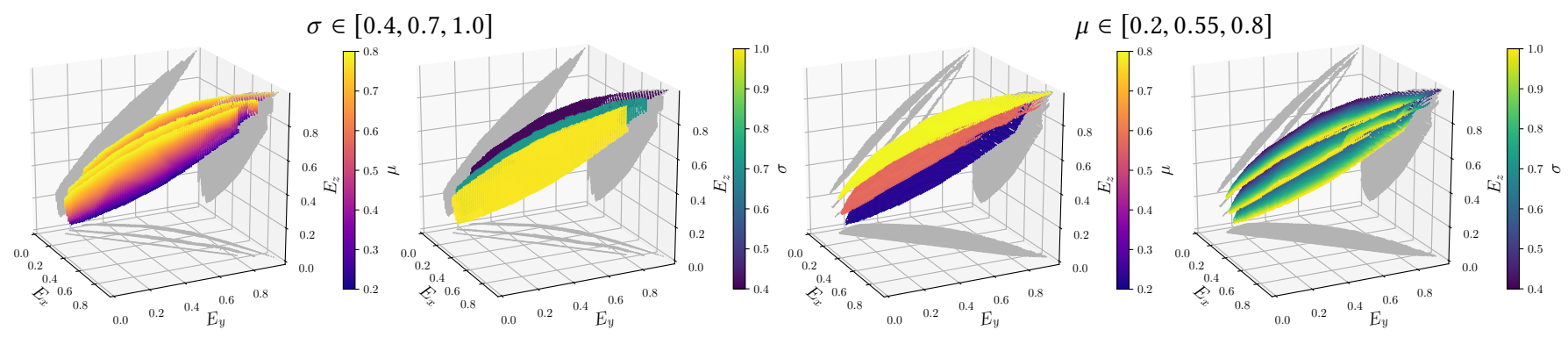

Fig. 15. Interpolation given by the material space sampling shown in Figure $13\left(\theta=45^{\circ}, k=8\right)$. The plot axes correspond the three orthogonal Young's moduli. For a three different values of $\sigma$ (left) and $\mu$ (right), we obtain three different curves of feasible spatial variations. 

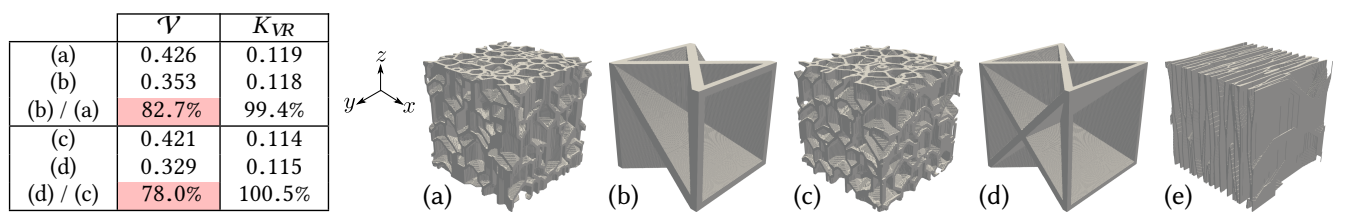

\begin{tabular}{|c|c|c|c|}
\cline { 2 - 4 } \multicolumn{1}{c|}{} & $\mathcal{V}$ & $E_{y}$ & $E_{z}$ \\
\hline (e) & 0.328 & 0.258 & 0.244 \\
$(\mathrm{~b})$ & 0.353 & 0.152 & 0.258 \\
$(\mathrm{e}) /(\mathrm{b})$ & $93.0 \%$ & $169.7 \%$ & $94.57 \%$ \\
\hline (d) & 0.329 & 0.199 & 0.199 \\
$(\mathrm{e}) /(\mathrm{d})$ & $99.7 \%$ & $129.6 \%$ & $122.6 \%$ \\
\hline
\end{tabular}

Fig. 16. We compare infills made of tessellations made of planes with polyhedral Voronoi diagrams. We set a minimum slope of $\theta=45^{\circ}$ for all tests. We give in the supplemental material the full expression of the homogenized elasticity tensors of each test. Left table: We determine the volume $\mathcal{V}$ required to achieve similar $K_{V R}$ values $( \pm 0.001)$. Since polyhedral Voronoi diagrams span a wide space of material, we select the closest sample (with respect to $K_{V R}$ ) in the database of Figure 12. However, note that other samples having similar $K_{V R}$ are possible. (a) and (c) correspond, respectively, to the closest match for (b) and (d). As seen in the third and sixth row, our infills require $20 \%$ more material for the same $K_{V R}$. Right table: When orthotropy is desired, for instance to mimic a laminate much stiffer in $y, z$ than in $x$, polyhedral Voronoi diagrams are better suited. As seen in the third row, for a lower volume (e) is significantly more stiff in $y$ than (b), while in the fifth row (e) is significantly stiffer in both $y, z$ than (d) for a similar volume.

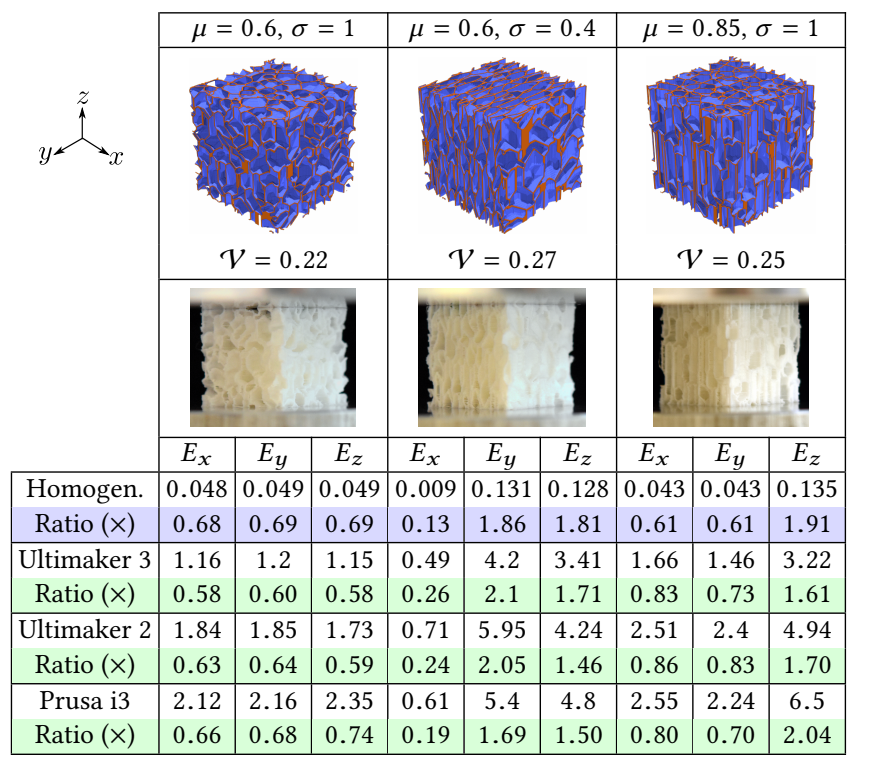

Fig. 17. Comparison between numerical homogenization (Young's moduli in the normalized range $[0,1])$ and experimental compression tests performed on an Instron 3345 testing machine. Experimental Young's moduli are given in megapascals (MPa). For all three tests we set $k=8$ and $\theta=45^{\circ}$, and we vary $\mu$ and $\sigma$. We have printed each sample (30 mm cubes) on three different printers (Ultimaker 3, and Ultimaker 2, and Prusa i3) using different rolls of filaments (Ninjatek Semiflex) in diameters of either $1.75 \mathrm{~mm}$ (Prusai3) or $2.85 \mathrm{~mm}$ (Ultimakers), with the same layer thickness $(0.2 \mathrm{~mm})$ and print speed $(15 \mathrm{~mm} / \mathrm{sec})$. We apply a compression test to each direction, to obtain $E_{x}, E_{y}$, and $E_{z}$. For comparison we compute ratios to the average value across all nine tests of the same printer (i.e. how much more rigid/flexible each is with respect to the average). As can be seen the ratios agree well overall. We provide in the supplemental material the full expression of the homogenized elasticity tensor of each compression test.

not been studied in the context of microstructures. Nevertheless, we provide here some elements of comparison.

A first important limitation of rhombic structures is that they cannot be graded spatially in orientation or anisotropy, due to their regular structure. Therefore, they offer significantly less control than our approach. Their density can be varied by subdivision, for

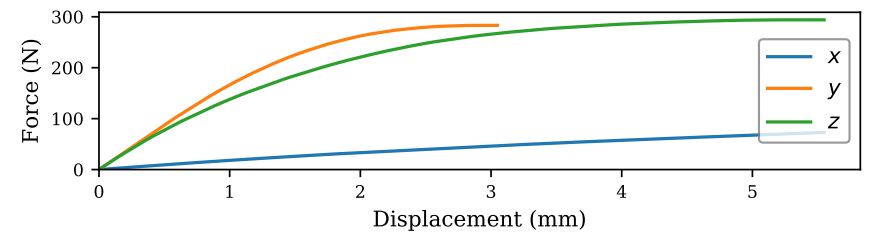

Fig. 18. Compression curve for the middle sample from Figure 17, printed on Prusai3. The material enters into the non-linear regime for the $y$ and $z$ axes when the load approaches $300 \mathrm{~N}$, it remains linear for the $x$ axis (low stiffness). We stopped the test for $y$ as the response flattened.

instance maximizing rigidity [Wu et al. 2016]. However, as illustrated in Figure 25, grading by subdivision introduces sudden jumps that impair the smoothness of the final elastic behavior.

Figure 16 compares the bulk modulus of the structures, for the two typical cases of using three or fours sets of parallel planes. Rhombic structures are $20 \%$ stiffer for a same volume, but this advantage vanishes if orthotropy is desired: our structures become significantly stiffer in the preferred directions for a same volume.

The rhombic structures, thanks to the use of straight lines, print slightly faster than our structures (14\% difference on a Prusai 3 on default settings, may vary with acceleration settings).

\section{APPLICATIONS}

In this section we present several designs modeled with our technique. The control fields are created either through a dedicated painting interface, or procedurally (we use a domain specific language for this purpose).

The designs we present are illustrations, demonstrating the versatility of our technique. To reveal the foam we print them without an external perimeter - this means that some small unsupported regions on the sides exist. In practice we observe no detrimental impact. Real designs could add an external thin skin where needed.

A striking difference between our designs and those produced with SLA/SLS is the ease of production - thanks to the use of FFF printers - as well as the robustness of the final part. In particular, parts printed with filaments having varying degrees of elasticity (Ninjatek SemiFlex and Cheetah) can flex very significantly without breaking. In our experience, foams are much more difficult to 


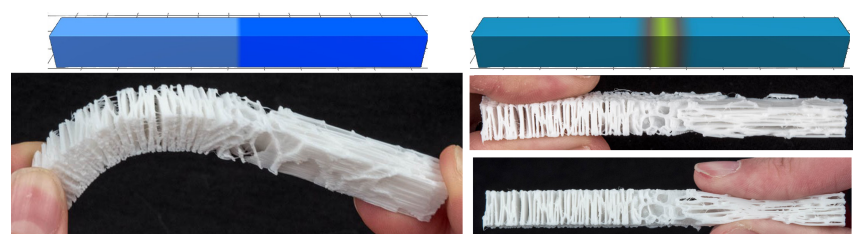

Fig. 19. Top row: Orientation field $\zeta$ (left) and anisotropy field $\sigma$ (right) Pictures, bottom row: The left side bends easily while the right side remains rigid. On the contrary, the right side can be compressed sideways (bottom image), while the left side is much more rigid (top image).
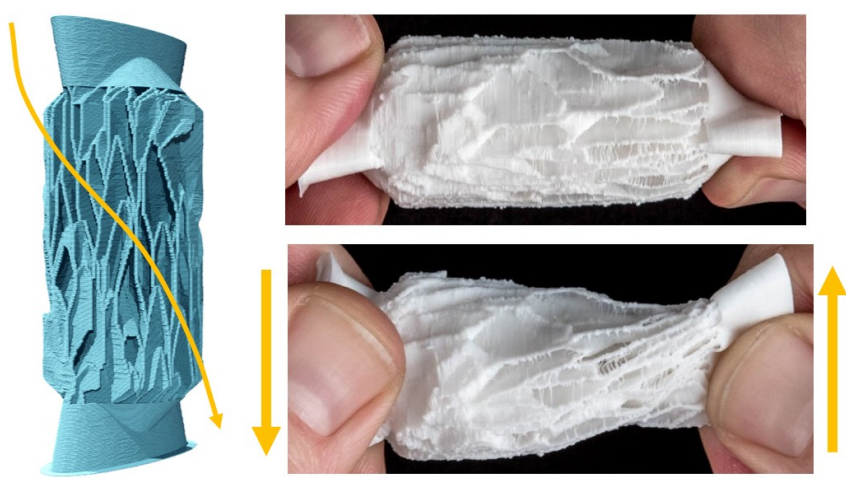

Fig. 20. Cylinder with a varying direction of orthotropy (from 0 to 90 degrees between the handles), vertically rigid but allowing rotation in one direction.

produce on SLA printers, require a careful choice of printer and parameters [Zhou 2015], and are more fragile after curing.

Our first example is a bar with varying orthotropy, shown in Figure 19. An abrupt change in orthotropy direction in the middle of the bar triggers very different behavior on both sides.

Our second example is a cylinder with a direction of orthotropy that rotates 90 degrees along the main axis, shown Figure 20. This creates an interesting case where, under rotation in the proper orientation, the cylinder twists and flatten across its section. It is otherwise rigid vertically.

Our third example is an illustration of a prosthetic finger, reproducing a result from [Martínez et al. 2016]. The two joints are obtained by a strong orthotropy aligned with the joint "axis." Density also varies between the outside (denser) and the inside of the bend. The result offers a preferred direction of flex that corresponds better to a natural joint, in particular it is harder to bend sideways (by orthotropy) or reverse (by density). See Figure 21.

Our fourth example is a wheel that can be mounted on an actual $\mathrm{RC}$ car (a Monster Beetle by Tamiya), see Figure 22. The wheel prints as a single part, is lighter than the original (109 $\mathrm{g}$ versus $130 \mathrm{~g})$, and deforms radially (shock absorbent). Of course, the design would have to be refined to act as an efficient tire. Our technique makes such applications accessible to hobbyists.

Our fifth example, Figure 23, illustrates how orthotropy can be arranged radially to achieve different properties in a pipe design.
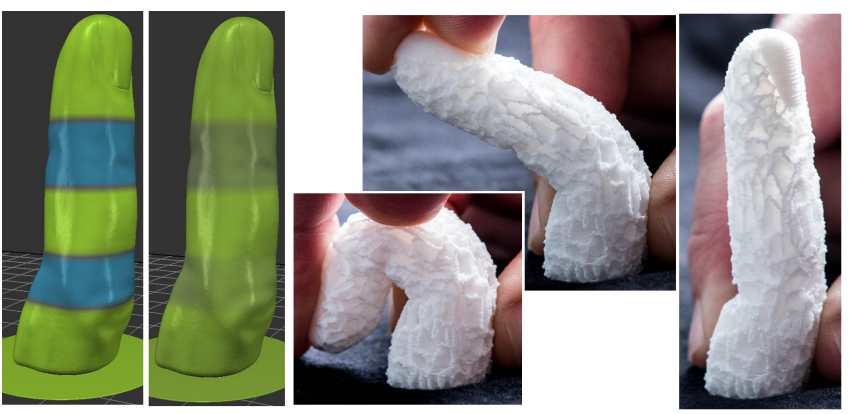

Fig. 21. The articulations of this finger prosthetic are obtained by spatially varying density and anisotropy. The design is very robust and easy to print: it uses a rubber-like filament (Cheetah by NinjaTek) printed on a standard FFF printer (Prusai3).
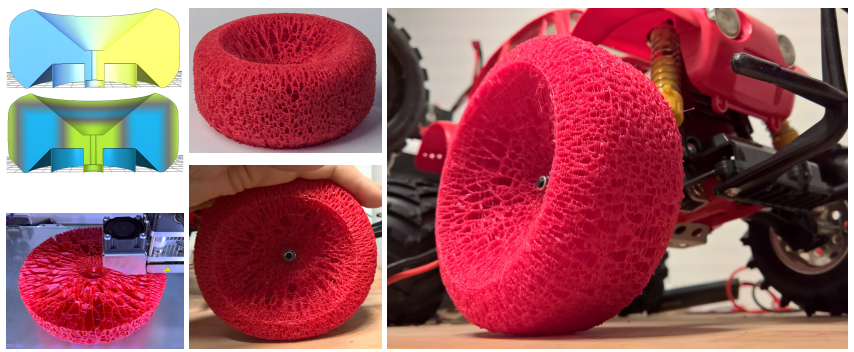

Fig. 22. Top left: Orientation field $\zeta$ (top) and density field (below). The anisotropy is constant at $\sigma=0.3$. Bottom left: Wheel being printed on an Ultimaker 2 with Semiflex filament at $0.3 \mathrm{~mm}$ thickness. The print took 19 hours. Middle column: The finished wheel and its deformation under load. Right: Ready to run!
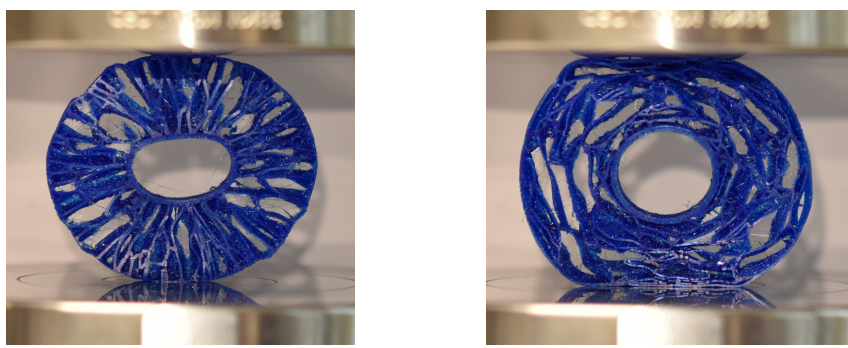

Fig. 23. An application to pipe design. The left design transfers forces between the inner and outer pipes, while the right one protects the inner pipe from external forces. Both designs differ only by the orthotropy angle.

Our final example is a shoe sole with controlled elasticity, shown in Figure 24. By modifying density, angle and anisotropy we are able to control both the "softness" and "bending" of the sole. While this is a purely illustrative example, we hope our method will help produce orthopaedic footwear at much lower price points than those produced on high-end SLS/SLA printers. 


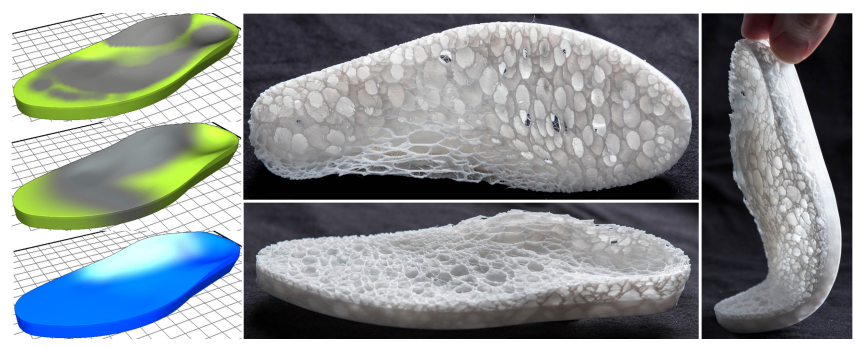

Fig. 24. A 3D printed shoe sole. Left: Control fields used on the model, density (top), orthotropy strength (middle) and angle (bottom). Right: Printed shoe, top, side and bending. The shoe is printed without any skin to reveal the foam structure.

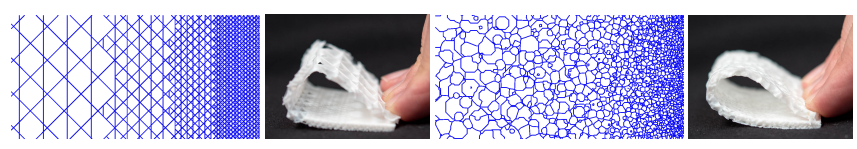

Fig. 25. Compared to subdividing rhombic infill (left), Voronoi polyhedral diagrams produce a smoother grading (right) without marked transitions, triggering a smoother elastic behavior. Both structures use the same amount of material. Ours printed $14 \%$ slower due to a more complex geometry.

\section{LIMITATIONS, FUTURE WORK}

To the best of our knowledge, the presented microstructures form the first metamaterial that is well suited for FFF and offers a wide range of controllable elastic behaviors. Their properties can be graded following custom control fields, while still satisfying strict guarantees on manufacturability.

There are however a number of limitations and areas of improvement. In terms of overall rigidity (bulk modulus), our structures are less effective than plane tessellations (see Figure 16). It remains an open question whether there exists other polyhedral distances improving bulk modulus, while enforcing fabrication constraints.

The geometry of the cone distance that we use entails a material with a orthotropy axis that can not be moved aways from the vertical axis. This lost degree of freedom may be desirable in some application. We believe further exploration of the space of polyhedral distances could let us recover this degree of freedom even while respecting the fabricability constraints of FFF-like processes.

The stochastic geometry has an impact on printing speed (about $15 \%$ slower than straight line infill on our printers). This possibly could be mitigated by optimizing the traversal of the paths; our slicer currently uses a simple "go to next closest path" heuristic.

A minor issue related to design is that the point sites are not always close to the centroid of the Voronoi cell. Therefore, at low densities and high anisotropy, there can be an offset between the control field and its actual effect of the structure. It would be interesting to compensate for this.

Finally, while we efficiently extract slices, we did not investigate interactive visualization of the microstructures - one possible approach is ray-marching. Efficient ray-traversal of the structures is an interesting topic for future work.

\section{CONCLUSIONS}

By carefully choosing the distance functions used to model Voronoi diagrams, we are able to produce stochastic closed-cell foams that enforce all manufacturability constraints on continuous deposition processes. We proposed a parameterized polyhedral distance based on cones, that provides good correlation between well understood geometric properties and observed elastic behaviors.

We envision that our method could also be helpful in optimizing periodic microstructures [Panetta et al. 2015; Schumacher et al. 2015] since it provides a space of geometric structures that are certain to be fabricable. It may also be interesting to use it as a replacement in approaches that optimize Voronoi diagrams for additive manufacturing, such as the work of Lu et al. [2014].

There is no direct way to interpret our structures as open-cell foams. The Voronoi edges could be considered but they do not enforce fabrication constraints. Therefore, our technique is complementary to existing works targeting powder and resin based systems, as it answers the need for parameterized metamaterials applicable to continuous deposition.

\section{ACKNOWLEDGMENTS}

We thank Noémie Vennin and Jimmy Etienne for their help in designing and printing results. This work was supported by $\mathrm{ERC}^{5}$ grant ShapeForge (StG-2012-307877), ANR ${ }^{6}$ MuFFin (ANR-17-CE100002), Région Lorraine and FEDER. ${ }^{7}$

\section{REFERENCES}

Jean-Daniel Boissonnat, Micha Sharir, Boaz Tagansky, and Mariette Yvinec. 1995. Voronoi Diagrams in Higher Dimensions Under Certain Polyhedral Distance Functions. In Proceedings of the Eleventh Annual Symposium on Computational Geometry. 79-88. https://doi.org/10.1145/220279.220288

Guylaine Boittin, Daniela Garajeu, Alice Labé, Hervé Moulinec, Fabrice Silva, and Pierre Suquet. 2014. CraFT. http://craft.lma.cnrs-mrs.fr/

Guylaine Boittin, Pierre-Guy Vincent, Hervé Moulinec, and Mihai Gărăjeu. 2017. Numerical simulations and modeling of the effective plastic flow surface of a biporous material with pressurized intergranular voids. Computer Methods in Applied Mechanics and Engineering 323, Supplement C (2017), $174-201 . \quad$ https: //doi.org/10.1016/j.cma.2017.05.004

L. Paul Chew and Robert L. (Scot) Dyrsdale, III. 1985. Voronoi Diagrams Based on Convex Distance Functions. In Proceedings of the First Annual Symposium on Computational Geometry. 235-244. https://doi.org/10.1145/323233.323264

A.G. Corbalan, M. Mazon, and T. Recio. 1996. Geometry of bisectors for strictly convex distances. International fournal of Computational Geometry \& Applications 06, 01 (1996), 45-58. https://doi.org/10.1142/S0218195996000046

Lorna J Gibson and Michael F Ashby. 1999. Cellular solids: structure and properties. Cambridge university press.

Richard Hill. 1952. The elastic behaviour of a crystalline aggregate. Proceedings of the Physical Society. Section A 65, 5 (1952), 349.

Samuel Hornus, Sylvain Lefebvre, Jérémie Dumas, and Frédéric Claux. 2016. Tight Printable Enclosures and Support Structures for Additive Manufacturing. In Proceedings of the Eurographics Workshop on Graphics for Digital Fabrication (GraDiFab '16). 11-21. https://doi.org/10.2312/gdf.20161074

Christian Icking and Lihong Ha. 2001. A Tight Bound for the Complexity of Voronoi Diagrams Under Polyhedral Convex Distance Functions in 3D. In Proceedings of the Thirty-third Annual ACM Symposium on Theory of Computing. 316-321. https: //doi.org/10.1145/380752.380815

Christian Icking, Rolf Klein, Ngoc-Minh Lé, and Lihong Ma. 1995. Convex distance functions in 3-space are different. Fundamenta Informaticae 22, 4 (1995), 331-352. https://doi.org/10.3233/FI-1995-2242

Christian Icking, Rolf Klein, Lihong Ma, Stefan Nickel, and Ansgar Weißler. 2001. On bisectors for different distance functions. Discrete Applied Mathematics 109, 1 (2001), 139-161. https://doi.org/10.1016/S0166-218X(00)00238-9

\footnotetext{
${ }^{5}$ European Research Council

${ }^{6}$ Agence nationale de la recherche

${ }^{7}$ Fond européen de développement régional
} 
Alexandra Ion, Johannes Frohnhofen, Ludwig Wall, Robert Kovacs, Mirela Alistar, Jack Lindsay, Pedro Lopes, Hsiang-Ting Chen, and Patrick Baudisch. 2016. Metamaterial Mechanisms. In Proceedings of the 29th Annual Symposium on User Interface Software and Technology (UIST '16). 529-539. http://doi.acm.org/10.1145/2984511.2984540

Rolf Klein and Derick Wood. 1988. Voronoi diagrams based on general metrics in the plane. Springer Berlin Heidelberg, 281-291. https://doi.org/10.1007/BFb0035852

M. Leary, M. Babaee, M. Brandt, and A. Subic. 2013. Feasible Build Orientations for Self-Supporting Fused Deposition Manufacture: A Novel Approach to SpaceFilling Tesselated Geometries. Advanced Materials Research 633 (2013), 148-168. https://doi.org/10.4028/www.scientific.net/AMR.633.148

Jusung Lee and Kunwoo Lee. 2017. Block-based inner support structure generation algorithm for $3 \mathrm{D}$ printing using fused deposition modeling. The International Journal of Advanced Manufacturing Technology 89, 5 (2017), 2151-2163. https: //doi.org/10.1007/s00170-016-9239-3

Mokwon Lee, Qing Fang, Joonghyun Ryu, Ligang Liu, and Deok-Soo Kim. 2018. Support-Free Hollowing for 3D Printing via Voronoi Diagram of Ellipses. Computer Aided Design 101 (2018), 23-36. https://arxiv.org/abs/1708.06577

Sylvain Lefebvre. 2015. 3D infilling: faster, stronger, simpler. http://sylefeb.blogspot.fr/ 2015/07/3dprint-3d-infilling-faster-stronger.html.

Xingchen Liu and Vadim Shapiro. 2016. Homogenization of material properties in additively manufactured structures. Computer-Aided Design 78, Supplement C (2016), 71-82. https://doi.org/10.1016/j.cad.2016.05.017

Marco Livesu, Stefano Ellero, Jonàs Martínez, Sylvain Lefebvre, and Marco Attene. 2017. From 3D Models to 3D Prints: An Overview of the Processing Pipeline. Computer Graphics Forum 36 (2017), 537-564.

Lin Lu, Andrei Sharf, Haisen Zhao, Yuan Wei, Qingnan Fan, Xuelin Chen, Yann Savoye Changhe Tu, Daniel Cohen-Or, and Baoquan Chen. 2014. Build-to-last: Strength to Weight 3D Printed Objects. ACM Trans. Graph. 33, 4, Article 97 (July 2014), 10 pages. https://doi.org/10.1145/2601097.2601168

Mathias H. Luxner, Juergen Stampfl, and Heinz E. Pettermann. 2007. Numerical simulations of 3D open cell structures - influence of structural irregularities on elastoplasticity and deformation localization. International fournal of Solids and Structures 44, 9 (2007), 2990 - 3003

Lihong Ma. 2000. Bisectors and Voronoi diagrams for convex distance functions. Ph.D. Dissertation. Fernuniversität, Fachbereich Informatik.

Jonàs Martínez, Jérémie Dumas, and Sylvain Lefebvre. 2016. Procedural Voronoi Foams for Additive Manufacturing. ACM Trans. Graph. 35, 4 (2016), 44:1-44:12. https://doi.org/10.1145/2897824.2925922

Jonàs Martínez, Haichuan Song, Jérémie Dumas, and Sylvain Lefebvre. 2017. Orthotropic k-nearest foams for additive manufacturing. ACM Transactions on Graphics 36, 4 (July 2017), 121:1-121:12. https://doi.org/10.1145/3072959.3073638

H. Martini and K.J. Swanepoel. 2004. The geometry of Minkowski spaces - A survey. Part II. Expositiones Mathematicae 22, 2 (2004), 93 - 144. https://doi.org/10.1016/ S0723-0869(04)80009-4

Sara McMains, Jordan Smith, Jianlin Wang, and Carlo Sequin. 2000. Layered Manufacturing of Thin-Walled Parts. In ASME Design Engineering Tech nical Conference, Baltimore, Maryland. arXiv:http://www.cs.berkeley.edu/ sequin/PAPERS/ASMEdetc00.pdf

Asla Medeiros e Sá, Vinícius Moreira Mello, Karina Rodriguez Echavarria, and Derek Covill. 2015. Adaptive voids. The Visual Computer 31, 6 (2015), 799-808. https //doi.org/10.1007/s00371-015-1109-8

Maher Moakher and Andrew N. Norris. 2006. The Closest Elastic Tensor of Arbitrary Symmetry to an Elasticity Tensor of Lower Symmetry. Fournal of Elasticity 85, 3 (2006), 215-263. https://doi.org/10.1007/s10659-006-9082-0

H. Moulinec and P. Suquet. 1998. A numerical method for computing the overall response of nonlinear composites with complex microstructure. Computer Methods in Applied Mechanics and Engineering 157, 1 (1998), 69-94. https://doi.org/10.1016/ S0045-7825(97)00218-1

Atsuyuki Okabe, Barry Boots, Kokichi Sugihara, and Sung Nok Chiu. 2009. Spatial Tessellations: Concepts and Applications of Voronoi Diagrams. John Wiley \& Sons.

Julian Panetta, Abtin Rahimian, and Denis Zorin. 2017. Worst-case Stress Relief for Microstructures. ACM Transactions on Graphics 36, 4 (2017), 122:1-122:16. https //doi.org/10.1145/3072959.3073649

Julian Panetta, Qingnan Zhou, Luigi Malomo, Nico Pietroni, Paolo Cignoni, and Denis Zorin. 2015. Elastic Textures for Additive Fabrication. ACM Trans. Graph. 34, 4 (2015), 135:1-135:12. https://doi.org/10.1145/2766937

Alexander Pasko, Oleg Fryazinov, Turlif Vilbrandt, Pierre-Alain Fayolle, and Valery Adzhiev. 2011. Procedural function-based modelling of volumetric microstructures. Graphical Models 73, 5 (2011), 165-181.

A.P. Roberts and E.J. Garboczi. 2002. Elastic properties of model random threedimensional open-cell solids. F. Mech. Phy. Solids 50, 1 (2002), 33 - 55.

Christian Schumacher, Bernd Bickel, Jan Rys, Steve Marschner, Chiara Daraio, and Markus Gross. 2015. Microstructures to Control Elasticity in 3D Printing. ACM Trans. Graph. 34, 4 (2015), 136:1-136:13. https://doi.org/10.1145/2766926
Ole Sigmund, Niels Aage, and Erik Andreassen. 2016. On the (non-)optimality of Michell structures. Structural and Multidisciplinary Optimization 54, 2 (2016), 361373. https://doi.org/10.1007/s00158-016-1420-7

TCT Ting and Tungyang Chen. 2005. Poisson's ratio for anisotropic elastic materials can have no bounds. Quart. F. Mech. Appl. Math. 58, 1 (2005), 73-82.

Kiril Vidimče, Szu-Po Wang, Jonathan Ragan-Kelley, and Wojciech Matusik. 2013 OpenFab: A Programmable Pipeline for Multi-material Fabrication. ACM Trans. Graph. 32, 4 (2013), 136:1-136:12.

W. Wang, Y. J. Liu, J. Wu, S. Tian, C. C. L. Wang, L. Liu, and X. Liu. 2017. SupportFree Hollowing. IEEE Transactions on Visualization and Computer Graphics (2017). https://doi.org/10.1109/TVCG.2017.2764462

Jun Wu, Charlie C.L. Wang, Xiaoting Zhang, and Rüdiger Westermann. 2016. Selfsupporting rhombic infill structures for additive manufacturing. Computer-Aided Design 80 (2016), 32-42. https://doi.org/10.1016/j.cad.2016.07.006

Yue Xie and Xiang Chen. 2017. Support-free interior carving for 3D printing. Visual Informatics 1, 1 (2017), 9-15. https://doi.org/10.1016/j.visinf .2017.01.002

Qingnan Zhou. 2015. A study in fabricating microstructures. https://medium.com/ 3d-printing-stories/a-study-in-fabricating-microstructures-part-1-f267d298326e.

Bo Zhu, Mélina Skouras, Desai Chen, and Wojciech Matusik. 2017. Two-Scale Topology Optimization with Microstructures. ACM Trans. Graph. 36, 5, Article 164 (July 2017), 16 pages. https://doi.org/10.1145/3095815 